Article

\title{
Whole Genome Sequence Analysis of Multidrug Resistant Escherichia coli and Klebsiella pneumoniae Strains in Kuwait
}

\author{
Ola H. Moghnia (D) and Nourah A. Al-Sweih * \\ Department of Microbiology, Faculty of Medicine, Kuwait University, Al-Safat 24923, Kuwait; \\ ola.maghnia@ku.edu.kw \\ * Correspondence: nourah.alsuwaih@ku.edu.kw; Tel.: +965-695-9733-7911
}

check for updates

Citation: Moghnia, O.H.; Al-Sweih, N.A. Whole Genome Sequence Analysis of Multidrug Resistant Escherichia coli and Klebsiella pneumoniae Strains in Kuwait. Microorganisms 2022, 10, 507. https://doi.org/10.3390/ microorganisms10030507

Academic Editor:

Andrew Shelenkov

Received: 19 January 2022

Accepted: 21 February 2022

Published: 25 February 2022

Publisher's Note: MDPI stays neutral with regard to jurisdictional claims in published maps and institutional affiliations.

Copyright: (c) 2022 by the authors. Licensee MDPI, Basel, Switzerland. This article is an open access article distributed under the terms and conditions of the Creative Commons Attribution (CC BY) license (https:// creativecommons.org/licenses/by/ $4.0 /)$.

\begin{abstract}
The spread of carbapenem-resistant Escherichia coli and Klebsiella pneumoniae is a global concern. The management of infections caused by multidrug resistance (MDR) isolates poses substantial clinical challenges in both hospitals and communities. This study aimed to investigate the genetic characteristics and variations of MDR E. coli and K. pneumoniae isolates. Bacterial identification and antibiotic susceptibility testing against 19 antibiotics were performed by standard methods. Whole genome sequencing (WGS) was carried out on eight carbapenem-resistant isolates using an Illumina MiSeq platform. The assembled draft genomes were annotated, then sequences were blasted against antimicrobial resistance (AMR) genes database. WGS detected several resistance genes mediating the production of $\beta$-lactamases, including carbapenems and extended-spectrum $\beta$-lactamase genes as ( $b l a_{\mathrm{OXA}-1 /-48}, b l a_{\mathrm{KPC}-2 /-29}, b l a_{\mathrm{CMY}-4 /-6}, b l a_{\mathrm{SHV}-11 /-12}, b l a_{\mathrm{TEM}-1}$, $b l a_{\mathrm{CTX}-\mathrm{M}-15}, b l a_{\mathrm{OKP}-\mathrm{B}}, b l a_{\mathrm{ACT}}$ and $\left.b l a_{\mathrm{EC}}\right)$. Furthermore quinolone resistance including oqxA/oq $x B$,

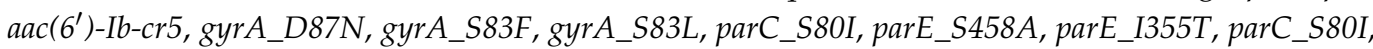
and qnrB1. In addition to aminoglycoside modifying enzymes genes (aph(6)-Id, aph(3")-Ib, aac(3)-IIa, $a a c\left(6^{\prime}\right)-I b, a a d A 1$, aadA2 and aadA5), trimethoprim-sulfamethoxazole (dfrA12/A14/A17 and sul1/sul2), tetracycline (tet $A$ and tet $B$ ), fosfomycin (fos $A$ and $u h p T \_E 350 Q$ ) resistance genes, while other genes were detected conferring chloramphenicol (floR, cat $A 2$, and efflux pump cmIA5), macrolides resistance $(m p h(A)$ and erm $(B)$, and quaternary ammonium efflux pump qacEdelta. Bleomycin and colistin resistance genes were detected as ble and $p m r B \_R 256 G$, respectively. Comprehensive analysis of MDR strains provided by WGS detected variable antimicrobial resistance genes and their precise resistance mechanism. WGS is essential for control and prevention strategies to combat the growing threat of AMR and the implementation of multifaceted interventions are needed.
\end{abstract}

Keywords: whole genome sequence; resistome; Klebsiella pneumoniae; Escherichia coli

\section{Introduction}

The escalating burden of antimicrobial resistance worldwide is substantial and is likely to grow [1]. The situation is complicated, since the development of resistance among Gram-negative bacteria is more rapid than Gram-positive bacteria [2], particularly antimicrobial-resistance strains (namely extended spectrum beta-lactamase [ESBL] and carbapenemase producers). The emergence of multidrug resistance (MDR) Enterobacterales such as Klebsiella pneumoniae and Escherichia coli is a well-known global health concern and possesses numerous resistance genes in its genome [3]. In healthcare settings, carbapenems are the drug of choice and are the most potent group of $\beta$-lactam antibiotics used to treat serious infections caused by ESBL-producing Enterobacterales and AmpC $\beta$-lactamase producers. They are frequently used for empiric therapy of life-threatening infections, such as sepsis [4,5]. The rising carbapenem resistance phenomenon in K. pneumoniae and E. coli is of particular concern, as this may lead to almost untreatable community-acquired infections [6]. The emergence and spread of MDR K. pneumoniae and E. coli isolates complicate the treatment of severe infections and threaten to create strains of bacteria resistant to currently 
available antimicrobial agents such as the $\beta$-lactam antibiotics [7]. The intensive use of antimicrobials in current practice may explain the expedition in developing and disseminating genes that encode resistance to $\beta$-lactam and other classes of antibiotics in K. pneumoniae and E. coli. Methods of discriminating and characterizing different K. pneumoniae and $E$. coli isolates are essential to targeting infection control resources. Several studies have been conducted in other countries detecting the prevalence of E. coli and K. pneumoniae carbapenem resistance. Prevalence rates ranged between 1 and $4 \%$ in Lebanon [8],3\% in Syria [9], 4\% in Iraq [10], 22.5\% in Jordan [11] and 2\% or less in African Arab countries such as Algeria, Libya, Morocco, Mauritania and Tunisia. However, reports from Egypt revealed the highest prevalence rate of $28 \%$ among E. coli and K. pneumoniae isolates [12,13]. A study showed increased prevalence rates of carbapenem resistance among K. pneumoniae than in E. coli isolates in Saudi Arabia [14]. In Kuwait, carbapenem resistance was detected in $3.4 \%$ among K. pneumoniae and E. coli strains isolated from the community [15]. To improve our understanding of transmission dynamics and antimicrobial resistance (AMR) gene diversity in outbreaks, it is recommended to use whole genome sequencing (WGS) of bacterial isolates, which is now well placed to become a gold standard in bacterial typing and to determine relatedness between strains, as it is highly discriminant in characterizing the complete genomic structure of isolates $[16,17]$. Advances in WGS assays have shown them to be a powerful tool for genotyping and the bacterial identification of clones in outbreak transmission with speed and depth of information [18]. Molecular dynamics reports of MDR isolates driving from the community at the whole genome level is limited. Previously, we identified the prevalence of genes mediating carbapenemase production in Enterobacterales isolates circulating in the community. We found that a relatively high number of carbapenem-resistant isolates harbored predominantly blaKPC among healthy individuals in community settings, which was uncommon in Kuwait and neighboring countries. In this study we sought to assess the use of whole genome sequencing (WGS) on selected E. coli and K. pneumoniae carbapenem-resistant isolates recovered from healthy individuals and patients, in order to understand their genomic diversity and genotypic presence of antimicrobial resistance.

\section{Materials and Methods}

\subsection{Bacterial Identification and Antimicrobial Susceptibility}

The study was carried out between May 2020 and October 2021 on eight MDR and confirmed carbapenem-resistant E. coli and K. pneumoniae isolates from rectal swabs recovered from healthy individuals and patients admitted to different hospitals in Kuwait. Representatives of carbapenem-resistant $E$. coli and K. pneumoniae isolates with carbapenemase-positive $(n=2)$ and carbapenemase-negative $(n=2)$ for each were chosen to gain further insight into the origin of genetic diversity and investigate resistance mechanisms. Sample collection and isolation methods were previously described $[19,20]$. Isolates were identified by VITEK 2 ID system (bioMérieux, Marcy-l'Etoile, France) and antimicrobial susceptibility testing was carried out to determine the minimum inhibitory concentration (MIC) in $\mu \mathrm{g} / \mathrm{mL}$ against 19 antibiotics using the Epsilometer test (E test) (bioMérieux, Marcy-l'Etoile, France), except for colistin, which was tested by agar dilution method as described previously [20]. Non-susceptible isolates to either one or more of the tested carbapenems with MIC of $>0.5 \mu \mathrm{g} / \mathrm{mL}$ for ertapenem or $>1 \mu \mathrm{g} / \mathrm{mL}$ for both imipenem and meropenem were selected for analysis. Antibiotic susceptibility results were interpreted according to the Clinical and Laboratory Standards Institute, 2020 breakpoints [21].

\subsection{Whole Genome Sequencing}

Genomic DNA from E. coli and K. pneumoniae were extracted using QIAamp ${ }^{\circledR}$ DNA Mini Kit (Qiagen, Hilden, Germany) according to the manufacturer's instructions. For each isolate, genomic DNA was quantified by the NanoDrop-800 spectrophotometer (Thermo Fisher Scientific, Wilmington, NC, USA) following the manufacturer's protocol. DNA extracts were fully sequenced by whole genome paired-end sequencing, Illumina data 
$(2 \times 150$ bp reads), including whole genome reconstruction, annotation, and antimicrobial resistance gene typing using the MiSeq sequencer (Illumina, San Diego, CA, USA). Accordingly, DNA libraries were prepared using Nextera XT library (Illumina, San Diego, CA, USA) targeted for each genome with $1 \mathrm{ng}$ genomic DNA per the manufacturer's recommendations. Briefly, tagment genomic DNA was simultaneously fragmented and then tagged with adapter sequences in a single step using Nextera transposome (Nextera XT DNA Library Preparation Kit, Illumina, San Diego, CA, USA). Tagmented DNA was then amplified using a limited-cycle (12-cycle) PCR program. To purify the library DNA, amplified DNA was cleaned up with AMPure XP beads. Then, Nextera libraries were quantified using Qubit and the size profile was determined on Agilent Technology 2100 Bioanalyzer using a high-sensitivity DNA chip (Agilent Technologies, Waldbronn, Germany). Various libraries can be sequenced with average fragment sizes that were generated from 828 to 1433 bases. Selected libraries for sequencing were normalized to $1 \mathrm{nM}$ and pooled. Pooling libraries combined equal volumes of normalized libraries in a single tube. Then, $1 \mathrm{nM}$ pooled library was diluted and heat-denatured before loading libraries for the sequencing run on a MiSeq sequencer (MiSeq reagent kit V2-300 cycles).

\subsection{Bioinformatics Analysis}

Paired-end reads from each E. coli and K. pneumoniae isolates were adapted, trimmed and quality-controlled using BBduk tool of the BBTools package (v38.59) (available online at https:/ /jgi.doe.gov / data-and-tools / software-tools/bbtools /bb-tools-user-guide/, accessed on 2 September 2021) to remove adapters, low-quality bases and ambiguous reads. Trimmed raw data were further assembled de novo using Spades (v3.13.1) algorithm to create a draft genome sequence for each isolate [22] and the data quality was checked with FastQC tool (v0.11.7) (available online at https: / / www.bioinformatics.babraham.ac. $\mathrm{uk}$ /projects / fastqc/, accessed on 2 September 2021). The assembled draft genomes were annotated by Prokka (v1.13.3) and CDS and protein sequences were extracted [23]. The WGS data were uploaded into the center for Genomic Epidemiology (available online at https: / / www.genomicepidemiology.org/services/index.html, accessed on 11 February 2022 and at https: / / pathogen.watch/, accessed on 11 February 2022) to detect virulence genes. The presence of insertion sequences was detected using ISFinder (available online at https:/ / www-is.biotoul.fr/blast.php/, accessed on 11 February 2022) to confirm their identity. The sequence types of isolates were determined based on WGS data (available online at https: / / cge.cbs.dtu.dk/services/mlst/, accessed on 11 February 2022).

\subsection{Antimicrobial Resistance Gene Typing}

Concurrently, each draft genome was screened for the presence of AMR genes. The most updated database of AMR genes downloaded from NCBI National Database of Antibiotic-Resistant Organisms (available online at https:/ / www.ncbi.nlm.nih.gov / pathogens / antimicrobial-resistance/, accessed 2 September 2021) was used as the reference for identifying the resistance genes present in the isolates. The scaffolds were blasted against all classes of AMR reference genes to infer the presence of all types of antibiotics. Sequenced raw data were blasted against retrieved genes using Standalone Blast (blastn, available online at https: / /blast.ncbi.nlm.nih.gov /Blast.cgi, accessed on 2 September 2021) [24].

\section{Results}

\subsection{Characteristics of Participants}

Four E. coli isolates were obtained from three females and one male. Of these, three Kuwaiti patients were admitted to three different hospitals, including Ibn Sina hospital (IBS) and Babtain hospital (Bab), located in the Capital (Al-Asimah) Governorate, in addition to Mubarak hospital (MK) in Hawali Governorate. The fourth isolate belonged to the healthy Filipino participant who lived in Farwaniya Governorate. However, four K. pneumoniae isolates were obtained from three males and one female. Two isolates belonged to Canadian and Kuwaiti patients who were admitted to MK in Hawali Governorate and Farwaniya 
hospital (FA) in Farwaniya Governorate, respectively. In addition, two isolates from healthy Indian participants who lived in Hawali and Farwaniya Governorates. All participants were aged between $1-80$ years old (Table 1 ).

Table 1. Demographic data of Escherichia coli and Klebsiella pneumoniae isolates obtained from patients and healthy participants.

\begin{tabular}{cccccccc}
\hline Strain ID & Isolates & Population & Nationality & Age Group & Gender & Governorate & Accession Number \\
\hline E1-112 & E. coli & Healthy & Filipino & $40-49$ & F & Farwaniya & SRX8356292 \\
E2-466 & E. coli & Patient & Kuwaiti & $50-59$ & M & Capital/(IBS) & SRX8356293 \\
E3-471 & E. coli & Patient & Kuwaiti & $70-79$ & F & Hawali/(MK) & SRX8356294 \\
E4-485 & E. coli & Patient & Kuwaiti & $20-29$ & F & Capital/(Bab) & SRX8356295 \\
K1-245 & K. pneumoniae & Healthy & Indian & $29-39$ & M & Hawali & SRX8344629 \\
K2-351 & K. pneumoniae & Healthy & Indian & $29-39$ & F & Farwaniya & SRX8344630 \\
K3-441 & K. pneumoniae & Patient & Canadian & $80-89$ & M & Hawali/(MK) & SRX8344631 \\
K4-500 & K. pneumoniae & Patient & Kuwaiti & $1-9$ & M & Farwaniya/(FA) & SRX8344632 \\
\hline
\end{tabular}

IBS: Ibn Sina hospital; MK: Mubarak hospital; Bab: Babtain hospital; FA: Farwaniya hospital.

\subsection{Genome Accession Numbers}

Raw genome data were submitted to the National Center for Biotechnology Information (NCBI) (available online at https://www.ncbi.nlm.nih.gov/sra/, accessed on 11 July 2021). The assembled genomic sequences of K. pneumoniae and E. coli isolates were deposited under the BioProjects (accession number PRJNA632581 and accession number PRJNA630112), respectively.

\subsection{Antibiotic Sensitivity Test}

Table 2 shows the antimicrobial susceptibility findings of eight isolates against 19 antimicrobial agents. All isolates were carbapenemase producers conferring resistant to ertapenem $(n=7)$, imipenem $(n=2)$, and meropenem $(n=2)$. A multi-drug resistance (MDR) profile, based on resistance to at least one agent in three or more antimicrobial classes was detected in all isolates. E. coli strains E1-112, E2-466, E3-471, and E4-485 were resistant to 8, 15, 13 and 17 antibiotics, respectively. While K. pneumoniae strains K1-245, K2-351, K3-441, and K4-500 were resistant to 3, 4, 13 and 12 antibiotics, respectively. The number of resistant isolates against 19 antibiotics are as following; $\beta$-lactams [ampicillin $(n=7)$, amoxicillin-clavulanic acid $(n=5)$, piperacillin $(n=5)$ ]; monobactam [aztreonam $(n=5)$ ]; cephalosporins [cephalothin $(n=6)$, cefepime $(n=4)$, cefotaxime $(n=6)$, cefoxitin $(n=4)$, ceftazidime $(n=6)$, ceftriaxone $(n=5)$ and cefuroxime $(n=6)]$; ciprofloxacin $(n=3)]$; colistin $(n=1)$; aminoglycosides [amikacin $(n=2)$ and gentamicin $(n=2)$ ] and tetracycline $(n=6)$.

\subsection{Whole Genome Sequencing and In Silico Data Analysis}

The isolates' final assembly, obtained by WGS, ranged from 52 to 138 contigs of $>500 \mathrm{bps}$ /sample in E. coli isolates with N50 values of between 124,277 and 560,222. A total of 52,138, 115 and 107 contigs representing 4,745,810;5,236,280; 5,369,672 and $5,463,758$ bases $(56 \%$ and $50 \% \mathrm{G}+\mathrm{C}$ ratio; $\mathrm{N} 50=560,222,124,277,183,231$ and 186,146) were obtained from assembled sequences of E. coli strains E1-112, E2-466, E3-471, and E4-485, respectively. In addition, 4417, 4845, 5015, 5149 CDS; 2, 3, 2, 3 rRNA; 69, 67, 73, 71 tRNA and $4489,4916,5091,5224 \mathrm{mRNA}$, respectively, were annotated for final contigs.

The isolates' final assembly ranged from 69 to 106 contigs of $>500 \mathrm{bps} /$ sample in K. pneumoniae isolates with N50 values of 139,608 and 375,813 . A total of 106, 80, 71 and 69 contigs representing 5,536,425; 5,4243,28,5,337,760 and 5,498,821 bases (56\% and 57\% $\mathrm{G}+\mathrm{C}$ ratio; $\mathrm{N} 50=139,608,272,188,375,813$ and 294,715) were obtained from assembled sequences of K. pneumoniae strains K1-245, K2-351, K3-441, and K4-500, respectively. Moreover, 5218, 5015, 4949, 5156 CDS; 2, 2, 1, 1 rRNA; 68, 74, 69, 70 tRNA; and 5289, 5092, 5020,5228 mRNA were annotated for final contigs. The main features of the E. coli and K. pneumoniae genome are shown in Tables 3 and 4. 
Table 2. Resistance pattern of Escherichia coli and Klebsiella pneumoniae isolates against selected antimicrobial agents.

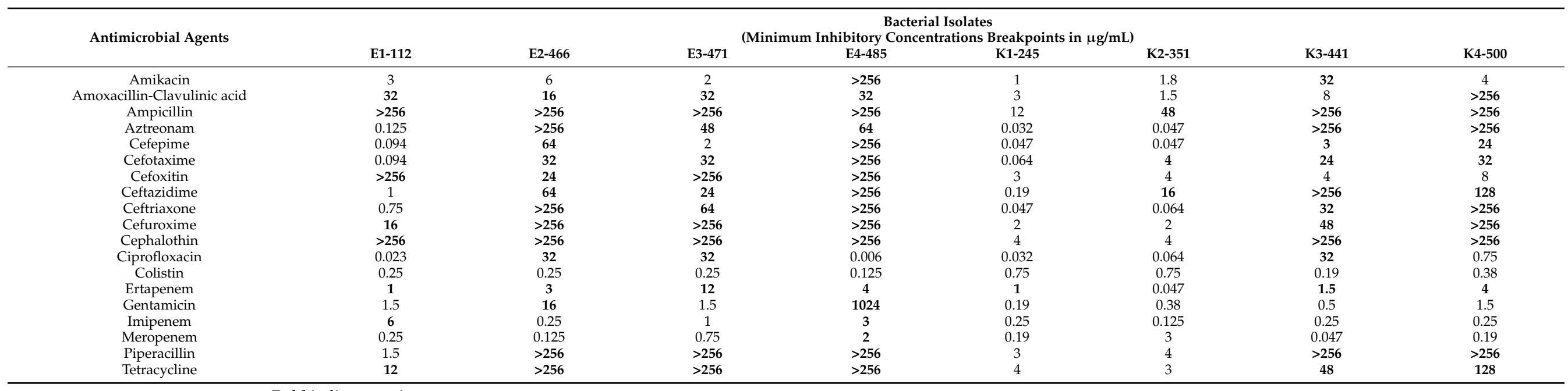

Bold indicates resistance.

Table 3. The summary statistics of the assembled draft genomes of Escherichia coli and Klebsiella pneumoniae isolates.

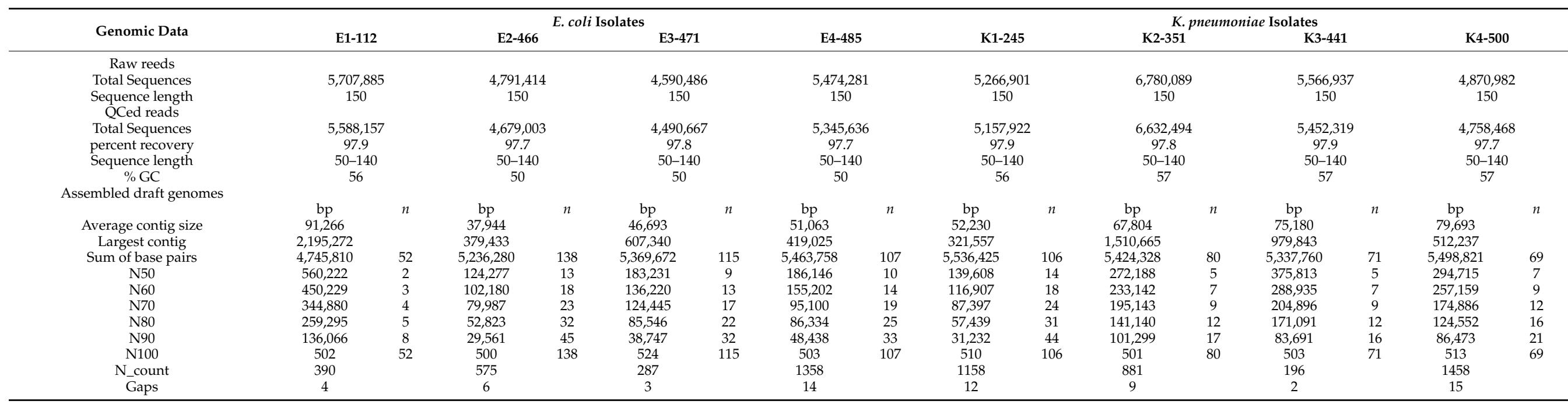


Table 4. Annotation of draft genome of Escherichia coli and Klebsiella pneumoniae isolates.

\begin{tabular}{|c|c|c|c|c|c|c|c|c|}
\hline \multirow{2}{*}{ Genomic Data } & \multicolumn{4}{|c|}{ E. coli Isolates } & \multicolumn{4}{|c|}{ K. pneumoniae Isolates } \\
\hline & E1-112 & E2-466 & E3-471 & E4-485 & K1-245 & K2-351 & K3-441 & K4-500 \\
\hline contigs/scaffolds & 52 & 138 & 115 & 107 & 106 & 80 & 71 & 69 \\
\hline bases & $4,745,810$ & $5,236,280$ & $5,369,672$ & $5,463,758$ & $5,536,425$ & $5,424,328$ & $5,337,760$ & $5,498,821$ \\
\hline gene & 4489 & 4916 & 5091 & 5224 & 5289 & 5092 & 5020 & 5228 \\
\hline CDS * & 4417 & 4845 & 5015 & 5149 & 5218 & 5015 & 4949 & 5156 \\
\hline tRNA * & 69 & 67 & 73 & 71 & 68 & 74 & 69 & 70 \\
\hline mRNA * & 4489 & 4916 & 5091 & 5224 & 5289 & 5092 & 5020 & 5228 \\
\hline rRNA * & 2 & 3 & 2 & 3 & 2 & 2 & 1 & 1 \\
\hline tmRNA * & 1 & 2 & 1 & 1 & 1 & 1 & 1 & 1 \\
\hline CRISPR * & - & 1 & 1 & 2 & - & - & - & - \\
\hline
\end{tabular}

* CDS coding sequence, tRNA: transfer RNA; mRNA: messenger RNA; rRNA: ribosomal RNA; tmRNA: transfermessenger RNA; CRISPR: Clustered Regularly Interspaced Short Palindromic Repeats.

\subsection{Antimicrobial Resistance Pattern}

Resistome analysis revealed the presence of various resistance genes that are demonstrated in Table 5. E. coli isolates carried the following $\beta$-lactamase genes $\left(\mathrm{E} 1-112 ; b l a_{A C T}\right.$ for AmpC $\beta$-lactamse enzymes) (E2-466; $\left.b l a_{E C}, b l a_{C T X-M-15}, b l a_{O X A-1}\right)$; (E3-471; bla $a_{K P C-2}$, $\left.b l a_{C M Y-4}, b l a_{T E M}\right)$ and $\left(\mathrm{E} 4-485 ; b l a_{E C}, b_{C M Y-6}, b l a_{C T X-M-15}\right)$. K. pneumoniae isolates harboured the following $\beta$-lactamase genes (K1-245; bla KPC-29, $\left.b l a_{O X A-48}\right)$; (K2-351; bla $\left.a_{S H V-11}\right) ;(\mathrm{K} 3-441$; bla $\left._{O K P-B}, b a_{S H V-12}\right)$ and $\left(\mathrm{K} 4-500 ;\right.$ bla $_{S H V-11}, b l a_{T E M-1}$, bla $\left._{C T X-M-15}, b l a_{O X A-1}\right)$. The presence of $\beta$-lactamase genes was related with sequences encoding for resistance to other classes of antibiotics in all the isolates: Three $E$. coli isolates conferring dihydrofolate reductase genes $d f r A$, including $d f r A 12 / 14 / 17$ alleles encoded trimethoprim resistance enzyme. Six isolates ( 3 E. coli and 3 K. pneumoniae) harbored sul1 and sul2 genes encoded sulfonamide resistance. The $m p h(A)$ and $\operatorname{erm}(B)$ genes were found in 3 E. coli and 2 E. coli isolates, respectively, encoding macrolide resistance. Both $t e t A$, tet $B$ genes encoding tetracycline resistance were detected in 2 K. pneumoniae and 2 E. coli, respectively. Aminoglycoside-modifying enzymes were detected in isolates with the following genes aph(6)-Id (1 E. coli and 2 K. pneumoniae), aph(3")-Ib (1 E. coli and 2 K. pneumoniae), aac $\left.6^{\prime}\right)$-Ib3 (1 E. coli), aac $\left(6^{\prime}\right)-I b$ (1 K. pneumoniae), aac(3)-IIa (1 E. coli), aadA1(1 K. pneumoniae), aadA2 (1 E. coli), and aadA5 (1 E. coli). Quinolone resistance was detected in 8 distinct genes, gyrA_D87N (2 E. coli and $1 \mathrm{~K}$. pneumoniae), gyrA_S83F (1 K. pneumoniae), gyrA_S83L (2 E.coli),parC_S80I (2 E. coli), parE_S458A (1 E. coli), parE_I355T (1 E. coli), parC_S80I (2 E. coli), qnrB1 (1 K. pneumoniae). For quinolone resistance, $a a c\left(6^{\prime}\right)-I b$-cr 5 gene was detected in 1 E. coli isolate. Furthermore, 1 E. coli and 4 K. pneumoniae isolates harbored multidrug efflux RND (Resistance-Nodulation-Division) transporter periplasmic adaptor subunit oqxA and multidrug efflux RND transporter permease subunit oqxB. For colistin resistance, $p m r B \_R 256 G$ gene was detected in 1 K. pneumoniae isolate. Six isolates ( $2 \mathrm{E}$. coli and $4 \mathrm{~K}$. pneumoniae) carried fos $A$ gene and $2 \mathrm{E}$. coli isolates carried $u h p T_{-} E 350 Q$ that conferred resistance to fosfomycin. Three $E$. coli isolates carried the cyaA_S352T gene that conferred resistance to fosmidomycin. Resistance genes cat $A 2$ in $1 \mathrm{~K}$. pneumoniae isolate and floR gene in $1 \mathrm{E}$. coli isolate were found to confer resistance to phenicol. The presence of ble gene detected in $1 \mathrm{E}$. coli isolate is responsible for bleomycin resistance. Three $E$. coli isolates carried quaternary ammonium compound efflux pump SMR transporter qacE delta1, and a single K. pneumoniae isolate harbored two efflux pumps: chloramphenicol efflux MFS transporter $c m l A 5$ gene and qacE delta1. Virulence genes were elucidated in E.coli isolates, E2-466 harbored enteroaggregative immunoglobulin repeat protein (air), outer membrane hemin receptor (chuA), colicin ia (cia), salmonella HilA homolog (eilA), siderophore receptor $(f y u A)$, glutamate decarboxylase ( $g a d)$, heat-resistant agglutinin ( $\mathrm{hra}$ ), high molecular weight protein 2 non-ribosomal peptide synthetase (irp2), capsule polysaccharide export inner-membrane protein $(\mathrm{kps} E)$, polysialic acid transport protein; group 2 capsule ( $\left.k p s M I I \_K 5\right)$, tellurium ion resistance protein (terC), and outer membrane protein complement resistance (traT). E3-471 isolate harbored the following genes: air, chuA, colicin ib (cib), gad, long polar fimbriae (lpfA), terC, traT, uropathogenic specific protein (usp), and fimbrial protein $(y f c V)$. E4-485 isolate carried the following: (air, chuA, eilA, fyuA, gad, 
irp2, increased serum survival (iss), aerobactin synthetase (iucC), ferric aerobactin receptor (iutA), capsule polysaccharide export inner-membrane protein ( $k p s E)$, lpfA, outer membrane protease (protein protease 7) (ompT), major pilin subunit F16 (papA_fsiA_F16), outer membrane usher $\mathrm{P}$ fimbriae ( $\mathrm{papC}$ ), secreted autotransporter toxin (sat), plasmid-encoded enterotoxin $(\operatorname{sen} B)$, and iron transport protein $(s i t A), \operatorname{ter} C$, and $\operatorname{traT})$. All K. pneumoniae isolates harbored wzi104, capsular locus (K locus) variant KL51, and (O locus) O1v2. Sequence types for E. coli isolates (E1-112, E2-466, E3-471 and E4-485) belonged to ST499, ST405, ST354 and ST69, respectively. Additionally, K. pneumoniae isolates (K1-245, K2-351, K3-441 and K4-500) belonged to ST3495, ST25, ST4743 and ST37, respectively. The insertion sequences as ISEc19, IS609, IS4, ISEC46 were detected in E. coli isolates and ISKpn74, ISKpn78, ISEc23 and ISKpn1 were detected in K. pneumoniae isolates.

Table 5. Types of resistance genes and mechanisms of Escherichia coli and Klebsiella pneumoniae isolates to different antimicrobial agents detected by WGS.

\begin{tabular}{|c|c|c|c|c|c|c|c|c|}
\hline \multirow{3}{*}{$\begin{array}{l}\text { Resistance to } \\
\text { Antibiotics }\end{array}$} & \multicolumn{4}{|c|}{ Escherichia coli Isolates } & \multicolumn{4}{|c|}{ Klebsiella pneumoniae Isolates } \\
\hline & \multicolumn{8}{|c|}{ Antimicrobial Resistance Genes } \\
\hline & E1-112 & E2-466 & E3-471 & E4-485 & K1-245 & K2-351 & K3-441 & K4-500 \\
\hline Beta-lactam & blaACT & $\begin{array}{c}\text { blaEC } \\
\text { blaCTX-M-15 } \\
\text { blaOXA-1 }\end{array}$ & $\begin{array}{c}\text { blaKPC-2 } \\
\text { blaCMY-4 } \\
\text { blaTEM }\end{array}$ & $\begin{array}{c}\text { blaEC } \\
\text { blaCMY-6 } \\
\text { blaCTX-M-15 }\end{array}$ & $\begin{array}{l}\text { blaKPC- } 29 \\
\text { blaOXA-48 }\end{array}$ & blaSHV-11 & $\begin{array}{l}\text { blaOKP-B } \\
\text { blaSHV-12 }\end{array}$ & $\begin{array}{c}\text { blaSHV-11 } \\
\text { blaTEM-1 } \\
\text { blaCTX-M-15 } \\
\text { blaOXA-1 }\end{array}$ \\
\hline Fosfomycin & $\begin{array}{c}\text { fosA } \\
\text { uhpT_E350Q }\end{array}$ & & $\begin{array}{c}\text { fosA } \\
u h p T \_E 350 Q\end{array}$ & & fos $A$ & fos $A$ & fos $A$ & fos $A$ \\
\hline Aminoglycoside & & $\begin{array}{c}a a d A 5 \\
\operatorname{aac}(3)-I I a\end{array}$ & $\begin{array}{l}\text { aph(6)-Id } \\
\text { aph(3")-Ib } \\
\text { aadA2 }\end{array}$ & $a a c\left(6^{\prime}\right)-I b 3$ & $\begin{array}{l}\operatorname{aph}(6)-I d \\
\operatorname{aph}\left(3^{\prime \prime}\right)-I b\end{array}$ & & $\begin{array}{l}a a c\left(6^{\prime}\right)-I b \\
\text { aad } A 1\end{array}$ & $\begin{array}{l}\operatorname{aph}(6)-I d, \\
\operatorname{aph}\left(3^{\prime \prime}\right)-I b\end{array}$ \\
\hline Sulfonamide & & sul1 & sul2, sul1 & sul1 & sul1 & & sul1 & sul2 \\
\hline \multirow[t]{2}{*}{ Quinolone } & $\begin{array}{l}o q x A \\
o q x B\end{array}$ & $a a c\left(6^{\prime}\right)-I b-c r 5$ & & & $\begin{array}{l}o q x A \\
o q x B\end{array}$ & oq $x B 17$ oq $x A 10$ & $\begin{array}{l}\operatorname{oq} x A \\
\operatorname{oq} x B\end{array}$ & $\begin{array}{l}o q x A \\
o q x B\end{array}$ \\
\hline & & $\begin{array}{c}\text { gyrA_D87N } \\
\text { gyrA_S83L } \\
\text { parC_S80I } \\
\text { parE_S458A }\end{array}$ & $\begin{array}{c}\text { gyrA_D87N } \\
\text { gyrA_S83L } \\
\text { parE_I355T } \\
\text { parC_S80I }\end{array}$ & & & & $\begin{array}{l}\text { gyrA_D87N } \\
\text { gyrA_S83F }\end{array}$ & qnrB1 \\
\hline Phenicol & & & floR & & & & catA2 & \\
\hline $\begin{array}{l}\text { Chloramphenicol } \\
\text { efflux pump }\end{array}$ & & & & & & & cmlA5 & \\
\hline $\begin{array}{l}\text { Quaternary } \\
\text { ammonium } \\
\text { efflux pump }\end{array}$ & & qacEdelta1 & qacEdelta1 & qacEdelta1 & & & qacEdelta1 & \\
\hline Tetracycline & & $\operatorname{tet}(B)$ & & $\operatorname{tet}(B)$ & & & $\operatorname{tet}(A)$ & $\operatorname{tet}(A)$ \\
\hline Fosmidomycin & & cyaA_S352T & cyaA_S352T & cyaA_S352T & & & & \\
\hline Trimethoprim & & dfrA17 & dfrA12 & dfrA14 & & & & \\
\hline Macrolide & & $m p h(A)$ & $m p h(A) \operatorname{erm}(B)$ & $m p h(A) \operatorname{erm}(B)$ & & & & \\
\hline Bleomycin & & & & ble & & & & \\
\hline Colistin & & & & & & & & pmrB_R256G \\
\hline
\end{tabular}

\section{Discussion}

E. coli and K. pneumoniae are important human pathogens that cause a wide spectrum of clinical diseases. To our knowledge, this is the first study to report the WGS analysis of asymptomatic carriers of MDR isolates in community settings in Kuwait and neighboring countries. Exploring the presence of AMR colonization is exceedingly essential, as these carriers may act as reservoirs for transmission of MDR bacteria. Moreover, the numbers are underestimated due to the absence of surveillance studies and efficient screening methods of individuals and focus only on patients admitted to healthcare settings. The advantages of WGS over the traditional sequencing technology is the ability to generate millions of reads in a single run with low costs. In the present study, the wide distribution of antibiotic resistance genes among isolates, including $\beta$-lactamase genes, ESBL and other genes conferring resistance to fosfomycin, aminoglycoside, sulfonamide, quinolone, phenicol, tetracycline, fosmidomycin, trimethoprim, macrolide, bleomycin, and colistin, in addition to several efflux pumps. Interestingly, K. pneumoniae and E. coli isolates showed elevated resistance rates to nearly all the $\beta$-lactam tested and other multiple antibiotics. All isolates were MDR phenotypes that carried at least one $\beta$-lactamase gene and the MICs of carbapenem antibiotics supported the presence of resistance genes of these antibiotics. The prevalence of Gram-negative pathogens has practical importance, especially for treatment options. In line 
with the findings of an Italian study, $\beta$-lactamase characterization of carbapenem-resistant K. pneumoniae isolates demonstrated the preponderance of carbapenemase production as K. pneumoniae carbapenemase (KPC) variants (blaKPC-2, blaKPC-3) and blaOXA. In addition, ESBLs, which include the Cefotaximase-Munich (CTX-M), Temoneria (TEM), and Sulfhydryl Variable (SHV) families were detected [25]. Our findings showed that the most prevalent ESBLs belong to blaSHV-11/12, blaTEM-1, blaCTX-M-15, blaCMY-6 genes. Those isolates also co-harbored carbapenemases as well blaKPC variants, blaKPC-2 and blaKPC-29 in addition to oxacillinase carbapenemase (OXA) variants, as blaOXA-48 and blaOXA-1 were detected. WGS confirmed the presence of KPC variants in Kuwait hospitals and community as one E. coli isolate with ST354 obtained from a Kuwaiti patient admitted to Mubarak hospital. The other isolate was K. pneumoniae with ST3495, obtained from a healthy Indian; they both live in Hawali Governorate, unlike previous publications that reported the predominance of OXA and New Delhi metallo- $\beta$-lactamas (NDM) variants in our region. It should be noted that $K$. pneumoniae disseminates easily via person-to-person contact, and its pathogenicity is enhanced by the easy acquisition of $\beta$-lactamase encoding genes. Therefore, the co-evolution of carbapenem-resistant strains is potentially the most worrying possibility due to the emergence of invasive $K$. pneumoniae infections, leading to treatment challenges.

Another interesting finding in our study was detecting a variety of resistance genes, highlighting the presence of sul gene in 7 isolates and fos $A$ gene in 6 isolates. Our findings parallel a study that reported the increased prevalence rates of sul variants, primarily in Gram-negative bacteria, including E. coli strains isolated worldwide [26]. However, fos $A$ gene is frequently found in Gram-negative species, including K. pneumoniae and $P$. aeruginosa, contributing to intrinsic fosfomycin resistance and it was rarely identified in $E$. coli. This may be due to the presence of fos $A$ gene on the chromosome in many Gram-negative species, whereas it rarely exists on the E. coli chromosome [27]. However, our findings showed that fos A gene was detected in all tested K. pneumoniae and two E. coli isolates; one of these E. coli isolates is a KPC-producing carbapenemase. A study reported that fos $A 6$ detected in a fosfomycin-resistant $E$. coli strain was likewise mobilized from the chromosome of K. pneumoniae, depending on its high degree of resemblance with fos $A^{\mathrm{KP}}$ and its position on a transposon [28]. Therefore, chromosomal fos $A$ genes in Gram-negative bacterial species may act as a reservoir of fosfomycin resistance in species without fos $A$ gene, such as E. coli [29].

Genomic data can be applied to trace and identify unanticipated modes of transmission and types of resistance genes. In our study, colistin resistance was conferred in an MDR K. pneumoniae isolate harbored $p m r B \_R 256 G$ gene obtained from a Kuwaiti patient. Resistance to colistin was not detected phenotypically and the isolate was within the susceptible MIC range for colistin; this may enhance the dissemination of such resistant genes silently in the hospital, as well as in the community. Moreover, it is known that K. pneumoniae has a high degree of environmental stability and survival in hospital environments and utensils, despite rigorous cleaning and decontamination procedures [30]. If we had such information, this asymptomatic patient could have been subjected to further strict surveillance cultures to detect potential sources of colonization and/or might be placed on contact isolation to cease any probable silent transmission chain that may occur in the hospital. Aminoglycoside resistance rates in E. coli and K. pneumoniae have been described in many countries, leading to global concerns [31,32]. The mechanism of resistance to aminoglycosides mainly occurs due to the presence of aminoglycosidemodifying enzymes (AMEs) and their different ability to modify aminoglycosides. These enzymes include acetyltransferases (AACs), nucleotidyltransferases (ANTs), and phosphotransferases (APHs) were described in Enterobacterales [33]. In our findings, MICs for gentamicin and/or amikacin showed high-level resistance in 3 isolates expressing various aminoglycosides gene as E4-485, aac (6 $\left.6^{\prime}\right)$-Ib3 gene; E2-466, aad and aac(3)-IIa genes and $\mathrm{K} 3-441, a a c\left(6^{\prime}\right)-I b$ and $a a d A 1$ genes. However, 3 isolates were susceptible to aminoglycosides tested found to carry variety of aminoglycosides resistance genes including E3-471, 
aph(6)-Id, aph(3")-Ib, aadA2; K1-245, aph(6)-Id, aph(3")-Ib and K4-500, aph(6)-Id, aph(3")-Ib. It should be noted that other types of aminoglycosides such as streptomycin, kanamycin, netilmicin, and tobramycin were not tested in our study, which may cause the presence of resistance genes in those isolates. Quinolone resistance was detected in 4 isolates; of these 3 isolates showed elevated MIC value to ciprofloxacin (MIC $>23 \mu \mathrm{g} / \mathrm{mL}$ ) and a great variety of quinolone-resistance determining regions (QRDRs) genes were detected. DNA gyrase (gyrA) mutations encoding the amino acid substitutions S83L, 83F and D87N were detected in $2 \mathrm{E}$. coli and $1 \mathrm{~K}$. pneumoniae isolates. These substitutions have been associated with quinolone resistance for $E$. coli and confer higher resistance levels than any other substitutions in the QRDRs, similar to that reported elsewhere [34], in addition to mutations causing the substitution in topoisomerase IV, parC_S80I, parE_S458A and parE_I355T. Another interesting finding is the presence of ciprofloxacin-sensitive K. pneumoniae that harboured plasmid-mediated quinolone resistance (PMQR) gene and expressed by the mediation of $q n r$ gene, namely as $q n r B 1$. This gene produces a pentapeptide protein that protects targeted enzymes from the effect of quinolones. Additionally, the presence of another method involves a mutation of $a a c\left(6^{\prime}\right)-I b-c r 5$ gene in one E. coli isolate, which confers resistance to quinolones by acetylation. A similar finding to that described by Magesh et al., 2011 reported the presence of multidrug-resistant K. pneumoniae isolates harboring $a a c\left(6^{\prime}\right)-1 b$-cr mutant gene in India [35]. The detection of efflux pumps belonging to $o q x A$ and $o q x B$ in our isolates may explain the mechanism of resistance. It has been reported as one of the main mechanisms of plasmid-mediated quinolone resistance that reduces the concentrations of quinolones intracellularly [36]. All these detected mechanisms are considered major contributors to the intrinsic and acquired multidrug resistance in Gram-negative bacteria. Our data showed that WGS determine accurately the exact mechanisms of antibiotic resistance in Enterobacterales. WGS platforms are rapidly spreading in clinical diagnostic laboratories that make genomic data more accessible and easily used in routine clinical settings, due to the lowering of turnaround time. Updates in DNA sequence techniques, plasmid conjugation and gene cloning should greatly improve our understanding of the spread and evolution of resistant strains. A limitation of the study was the requirement of expertise for bioinformatics analysis of data.

\section{Conclusions}

Our findings showed that whole genome sequencing and its in-depth analysis can accurately reconstruct the molecular characterization of isolates. The global spread of MDR K. pneumoniae and E. coli is a worrying phenomenon and close inspection to avoid their spread is strongly warranted. The clinical application of WGS could improve the surveillance of alert MDR pathogens by overcoming the limitation of analyzing only a small part of the genome and providing more rapid management and controlling the emergence of new antibiotic-resistant strains and their evolution. In view of the changing epidemiology of MDR clones, as demonstrated in this study and other reports in the literature, it is recommended in the future to perform active surveillance and to monitor any changes in resistant patterns, carriage of genes for virulence factors and prevalent clones.

Author Contributions: Conceptualization, O.H.M. and N.A.A.-S.; methodology O.H.M.; software, O.H.M.; validation, O.H.M. and N.A.A.-S.; formal analysis, O.H.M.; investigation, O.H.M.; resources, O.H.M.; data curation, O.H.M.; writing-original draft preparation, O.H.M.; writing-review and editing, O.H.M. and N.A.A.-S.; visualization, O.H.M.; supervision, N.A.A.-S. project administration, O.H.M. and N.A.A.-S. All authors have read and agreed to the published version of the manuscript.

Funding: This research received no external funding.

Institutional Review Board Statement: The study was conducted according to the guidelines of the Declaration of Helsinki, and approved by the Ethical Committee on Research Authorizations approved the research license for this study and the consent procedure for the Protection of Human Subjects in Research of Health Sciences Center, Kuwait University (No.299/2015).

Informed Consent Statement: Informed consent was obtained from all subjects involved in the study. 
Data Availability Statement: Genomic sequences of K. pneumoniae isolates have been deposited in the BioProject database, (available online at http:/ / www.ncbi.nlm.nih.gov/bioproject/632581, accessed on 1 July 2021); BioSample accessions including SAMN14913414, SAMN14913415, SAMN14913416, and SAMN14913417. Genomic sequences of E. coli isolates have been deposited in the BioProject database (available online at https:/ / www.ncbi.nlm.nih.gov/sra/PRJNA630112, accessed on 1 July 2021); BioSample accessions including SAMN14943485, SAMN14943486, SAMN14943487, and SAMN14943488.

Acknowledgments: We thank Mohd Wasif Khan for his technical assistance in data submission to the NCBI database.

Conflicts of Interest: The authors declare no conflict of interest.

\section{References}

1. Li, B.; Webster, T.J. Bacteria antibiotic resistance: New challenges and opportunities for implant-associated orthopedic infections. J. Orthop. Res. Off. Publ. Orthop. Res. Soc. 2018, 36, 22-32. [CrossRef]

2. Tan, T.T. "Future" threat of gram-negative resistance in Singapore. Ann. Acad. Med. Singap. 2008, 37, 884-890.

3. Paterson, D.L. Resistance in gram-negative bacteria: Enterobacteriaceae. Am. J. Infect. Control 2006, 34, S20-S73. [CrossRef]

4. Bush, K.; Fisher, J.F. Epidemiological expansion, structural studies, and clinical challenges of new $\beta$-lactamases from gramnegative bacteria. Annu. Rev. Microbiol. 2011, 65, 455-478. [CrossRef]

5. Patrier, J.; Timsit, J.F. Carbapenem use in critically ill patients. Curr. Opin. Infect. Dis. 2020, 33, 86-91. [CrossRef]

6. Tängdén, T.; Giske, C. Global dissemination of extensively drug-resistant carbapenemase-producing Enterobacteriaceae: Clinical perspectives on detection, treatment and infection control. J. Intern. Med. 2015, 277, 501-512. [CrossRef]

7. Boucher, H.W.; Talbot, G.H.; Bradley, J.S.; Edwards, J.E.; Gilbert, D.; Rice, L.B.; Scheld, M.; Spellberg, B.; Bartlett, J. Bad bugs, no drugs: No ESKAPE! An update from the Infectious Diseases Society of America. Clin. Infect. Dis. Off. Publ. Infect. Dis. Soc. Am. 2009, 48, 1-12. [CrossRef]

8. Chamoun, K.; Farah, M.; Araj, G.; Daoud, Z.; Moghnieh, R.; Salameh, P.; Saade, D.; Mokhbat, J.; Abboud, E.; Hamze, M.; et al. Surveillance of antimicrobial resistance in Lebanese hospitals: Retrospective nationwide compiled data. Int. J. Infect. Dis. 2016, 46, 64-70. [CrossRef]

9. Al-Assil, B.; Mahfoud, M.; Hamzeh, A.R. Resistance trends and risk factors of extended spectrum $\beta$-lactamases in Escherichia coli infections in Aleppo, Syria. Am. J. Infect. Control 2013, 41, 597-600. [CrossRef]

10. Aljanaby, A.A.J.; Alhasnawi, H. Research Article Phenotypic and Molecular Characterization of Multidrug Resistant Klebsiella pneumoniae Isolated from Different Clinical Sources in Al-Najaf Province-Iraq. Pak. J. Biol. Sci. 2017, 20, 217-232. [CrossRef]

11. Badran, E.F.; Qamer Din, R.A.; Shehabi, A.A. Low intestinal colonization of Escherichia coli clone ST131 producing CTX-M-15 in Jordanian infants. J. Med. Microbiol. 2016, 65, 137-141. [CrossRef] [PubMed]

12. Abdallah, H.; Wintermans, B.; Reuland, E.; Koek, A.; Al Naiemi, N.; Ammar, A.; Mohamed, A.A.; Vandenbroucke-Grauls, C.M.J.E. Extended-spectrum $\beta$-lactamase-and carbapenemase-producing Enterobacteriaceae isolated from Egyptian patients with suspected blood stream infection. PLoS ONE 2015, 10, e0128120-e0128127. [CrossRef] [PubMed]

13. Wassef, M.; Abdelhaleim, M.; AbdulRahman, E.; Ghaith, D. The Role of OmpK35, OmpK36 Porins, and Production of betaLactamases on Imipenem Susceptibility in Klebsiella pneumoniae Clinical Isolates, Cairo, Egypt. Microb. Drug Resist. 2015, 21, 577-580. [CrossRef] [PubMed]

14. Al-Agamy, M.H.; Shibl, A.M.; Hafez, M.M.; Al-Ahdal, M.N.; Memish, Z.A.; Khubnani, H. Molecular characteristics of extendedspectrum $\beta$-lactamase-producing Escherichia coli in Riyadh: Emergence of CTX-M-15-producing E. coli ST131. Ann. Clin. Microbiol. Antimicrob. 2014, 13, 4. [CrossRef]

15. Moghnia, O.H.; Rotimi, V.O.; Al-Sweih, N.A. Preponderance of bla $a_{\mathrm{KPC}}$-Carrying Carbapenem-Resistant Enterobacterales Among Fecal Isolates from Community Food Handlers in Kuwait. Front. Microbiol. 2021, 12, 737828. [CrossRef]

16. Lee, Y.; Kim, B.S.; Chun, J.; Yong, J.H.; Lee, Y.S.; Yoo, J.S.; Yong, D.; Hong, S.G.; D'Souza, R.; Thomson, K.S.; et al. Clonality and Resistome analysis of KPC-producing Klebsiella pneumoniae strain isolated in Korea using whole genome sequencing. BioMed Res. Int. 2014, 2014, 352862. [CrossRef]

17. Köser, C.U.; Ellington, M.J.; Peacock, S.J. Whole-genome sequencing to control antimicrobial resistance. Trends Genet. 2014, 30, 401-407. [CrossRef]

18. Didelot, X.; Bowden, R.; Wilson, D.J.; Peto, T.E.; Crook, D.W. Transforming clinical microbiology with bacterial genome sequencing. Nat. Rev. Genet. 2012, 13, 601-612. [CrossRef]

19. Moghnia, O.H.; Rotimi, V.O.; Al-Sweih, N.A. Evaluating Food Safety Compliance and Hygiene Practices of Food Handlers Working in Community and Healthcare Settings in Kuwait. Int. J. Environ. Res. Public Health 2021, 18, 1586. [CrossRef]

20. Moghnia, O.H.; Rotimi, V.O.; Al-Sweih, N.A. Monitoring antibiotic resistance profiles of faecal isolates of Enterobacteriaceae and the prevalence of carbapenem-resistant isolates among food handlers in Kuwait. J. Glob. Antimicrob. Resist. 2021, 25, 370-376. [CrossRef]

21. CLSI [Clinical and Laboratory Standard Institute]. Performance Standards for Antimicrobial Susceptibility Testing; 30th Informational Supplement CLSI Publication; M100-S30; CLSI: Wayne, PA, USA, 2020. 
22. Bankevich, A.; Nurk, S.; Antipov, D.; Gurevich, A.A.; Dvorkin, M.; Kulikov, A.S.; Lesin, V.M.; Nikolenko, S.I.; Pham, S.; Prjibelski, A.D.; et al. SPAdes: A new genome assembly algorithm and its applications to single-cell sequencing. J. Comput. Biol. A J. Comput. Mol. Cell Biol. 2012, 19, 455-477. [CrossRef] [PubMed]

23. Seemann, T. Prokka: Rapid prokaryotic genome annotation. Bioinformatics 2014, 30, 2068-2069. [CrossRef] [PubMed]

24. Camacho, C.; Coulouris, G.; Avagyan, V.; Ma, N.; Papadopoulos, J.; Bealer, K.; Madden, T.L. BLAST+: Architecture and applications. BMC Bioinform. 2009, 10, 421. [CrossRef] [PubMed]

25. Rimoldi, S.G.; Gentile, B.; Pagani, C.; Di Gregorio, A.; Anselmo, A.; Palozzi, A.M.; Lista, F. Whole genome sequencing for the molecular characterization of carbapenem-resistant Klebsiella pneumoniae strains isolated at the Italian ASST Fatebenefratelli Sacco Hospital, 2012-2014. BMC Infect. Dis. 2017, 17, 666. [CrossRef]

26. Jiang, H.; Cheng, H.; Liang, Y.; Yu, S.; Yu, T.; Fang, J.; Zhu, C. Diverse mobile genetic elements and conjugal transferability of sulfonamide resistance genes (sul1, sul2, and sul3) in Escherichia coli isolates from Penaeus vannamei and pork from large markets in Zhejiang, China. Front. Microbiol. 2019, 10, 1787. [CrossRef]

27. Ito, R.; Mustapha, M.M.; Tomich, A.D.; Callaghan, J.D.; McElheny, C.L.; Mettus, R.T.; Doi, Y. Widespread fosfomycin resistance in Gram-negative bacteria attributable to the chromosomal fosA gene. MBio 2017, 8, e00749-17. [CrossRef]

28. Guo, Q.; Tomich, A.D.; McElheny, C.L.; Cooper, V.S.; Stoesser, N.; Wang, M.; Sluis-Cremer, N.; Doi, Y. Glutathione-S-transferase FosA6 of Klebsiella pneumoniae origin conferring fosfomycin resistance in ESBL-producing Escherichia coli. J. Antimicrob. Chemother. 2016, 71, 2460-2465. [CrossRef]

29. Xu, H.; Miao, V.; Kwong, W.; Xia, R.; Davies, J. Identification of a novel fosfomycin resistance gene (fosA2) in Enterobacter cloacae from the Salmon River, Canada. Lett. Appl. Microbiol. 2011, 52, 427-429. [CrossRef]

30. Snitkin, E.S.; Zelazny, A.M.; Thomas, P.J.; Stock, F.; NISC Comparative Sequencing Program Group; Henderson, D.K.; Palmore, T.N.; Segre, J.A. Tracking a hospital outbreak of carbapenem-resistant Klebsiella pneumoniae with whole-genome sequencing. Sci. Transl. Med. 2012, 4, 148ra116. [CrossRef]

31. Caméléna, F.; Morel, F.; Merimèche, M.; Decousser, J.W.; Jacquier, H.; Clermont, O.; Darty, M.; Mainardis, M.; Cambau, E.; Tenaillon, O.; et al. IAME Resistance Group Genomic characterization of 16S rRNA methyltransferase-producing Escherichia coli isolates from the Parisian area, France. J. Antimicrob. Chemother. 2020, 75, 1726-1735. [CrossRef]

32. Nasiri, G.; Peymani, A.; Farivar, T.N.; Hosseini, P. Molecular epidemiology of aminoglycoside resistance in clinical isolates of Klebsiella pneumoniae collected from Qazvin and Tehran provinces, Iran. Infect. Genet. Evol. J. Mol. Epidemiol. Evol. Genet. Infect. Dis. 2018, 64, 219-224. [CrossRef] [PubMed]

33. Zhang, X.; Li, Q.; Lin, H.; Zhou, W.; Qian, C.; Sun, Z.; Lin, L.; Liu, H.; Lu, J.; Lin, X.; et al. High-Level Aminoglycoside Resistance in Human Clinical Klebsiella pneumoniae Complex Isolates and Characteristics of armA-Carrying IncHI5 Plasmids. Front. Microbiol. 2021, 12, 636396. [CrossRef] [PubMed]

34. Yoshida, H.; Bogaki, M.; Nakamura, M.; Yamanaka, L.M.; Nakamura, S. Quinolone resistance-determining region in the DNA gyrase gyrB gene of Escherichia coli. Antimicrob. Agents Chemother. 1991, 35, 1647-1650. [CrossRef] [PubMed]

35. Magesh, H.; Kamatchi, C.; Vaidyanathan, R.; Sumathi, G. Identification of plasmid-mediated quinolone resistance genes qnrA1, qnrB1 and aac(6')-1b-cr in a multiple drug-resistant isolate of Klebsiella pneumoniae from Chennai. Indian J. Med. Microbiol. 2011, 29, 262-268. [CrossRef]

36. Yuan, L.; Zhai, Y.J.; Wu, H.; Sun, H.R.; He, Z.P.; Wang, Y.B.; Pan, Y.S.; Kuang, N.N.; Hu, G.Z. Identification and prevalence of RND family multidrug efflux pump oqxAB genes in Enterococci isolates from swine manure in China. J. Med. Microbiol. 2018, 67, 733-739. [CrossRef] 